\title{
SALUD MENTAL, BIENESTAR E INCLUSIÓN SOCIAL EN PERSONAS ADULTAS HABITANTES DE CALLE
}

\author{
MENTAL HEALTH, WELL-BEING AND SOCIAL INCLUSION IN ADULT \\ STREET DWELLERS \\ Anny José Contreras Alfonso ${ }^{1 *}$, Gineth Daniela Navarrete Méndez ${ }^{1}$, Tania Gineth Ortiz Urrego ${ }^{1}$, \\ (iD) Viviana Carmenza Ávila Navarrete ${ }^{1}$ \\ anny.contrerasal@amigo.edu.co; gineth.navarreteme@amigo.edu.co; tania.ortizur@amigo.edu.co; \\ viviana.avilana@amigo.edu.co \\ ${ }^{1}$ Universidad Católica Luis Amigó, Bogotá, Colombia
}

*Correspondencia: Anny José Contreras Alfonso. Email: anny.contrerasal@amigo.edu.co

Recibido: 11.05.21 | Aprobado: 06.07.21

\section{RESUMEN}

El presente análisis, parte de una investigación acerca de temas relacionados con personas adultas habitantes de calle, por lo cual estos temas son: salud mental, inclusión social y bienestar social en la población nombrada anteriormente, por ende, se evidencia que existen muy pocas investigaciones respecto a dichos temas en la población a trabajar. Por tanto, se encuentran decretos y leyes, las cuales cobijan al habitante de calle, pero no se llevan a cabo con la población, lo cual da cuenta que hace falta mucha información e investigación al respecto. Se analiza la influencia que tiene la inclusión social en la salud mental y el bienestar social en las personas adultas habitantes de calle por medio de la revisión documental y de esta manera se llegue a una atención integral a nivel psicosocial, dado lo anterior se evidencia que no es mucha la relevancia que tiene la inclusión social en la salud mental y bienestar social en el habitante de calle, ya que no se trabaja con la población ningún aspecto referente a los temas anteriormente nombrados, lo cual evidencia que esta población es muy vulnerada y no tiene una buena salud mental al igual que las entidades no velan por su beneficio en todo sentido.

Palabras clave: bienestar, inclusión social, salud mental, habitante de calle.

\section{ABSTRACT}

The present analysis, part of an investigation about issues related to adult street dwellers, therefore these issues are: mental health, social inclusion and social well-being in the population named above, therefore it is evidenced that there is very little research regarding these issues in the population to work. Therefore, there are decrees and laws, which cover the street dweller but are not carried out with the population, which shows that a lot of information and research is needed in this regard. The influence that social inclusion has on mental health and social well-being in adult street dwellers is analyzed by means of a documentary review and in this way, comprehensive care is reached at the psychosocial level, given the foregoing it is evidenced that the relevance of social inclusion in mental health and social well-being in street dwellers is not very important, since no aspect regarding the aforementioned issues is worked with the population, which shows that this population is highly vulnerable and he does not have good mental health just as the entities do not look after his benefit in every sense.

Keywords: well-being, social inclusion, mental health, street dweller. 


\section{INTRODUCCIÓN}

Al hablar de salud mental se entiende que es un estado tanto de bienestar psicológico como emocional y social donde las personas pueden afrontar las diferentes presiones sociales presentes en la vida cotidiana, en el cual se pueden desenvolver sin llevar a cabo ningún tipo de afectación a su estado psicológico individual (Congreso de Colombia, 2013); sin embargo la salud mental no es prioridad para la población, debido a que se evidencia que es más importante tener una buena salud física que mental.

Por lo tanto, la salud mental en el habitante de calle no es totalmente importante, por lo cual se evidencia que esta población no es tomada en cuenta como parte de la sociedad, ya que no presenta algún tipo de bienestar tanto psicológico como emocional y social, dando cuenta que, si para la sociedad en general no es de vital importancia, para esta población tampoco es muy significativa.

Lo anterior da cuenta que la inclusión social en esta población no es evidente, ya que la misma lo que busca es que todos tengan los mismos derechos y que se genere una participación activa de los ciudadanos en las diferentes decisiones del país, lo anterior da cuenta que el bienestar del habitante de calle está relacionado con su estado de salud mental y la inclusión de la sociedad al mismo. De esta manera el habitante de calle no tiene una buena calidad de vida, sin embargo, la proyección de este análisis es ahondar algunas estrategias de inclusión en el habitante de calle partiendo de unas entidades que ayudan a incluir socialmente a esta población para que sea tomada en cuenta y no se siga estigmatizando como una población vulnerada, por tanto, el estado no proporciona derechos y deberes hacia el habitante de calle.

La problemática de esta población es de gran modo la exclusión social, por la cual ellos tienen que pasar, pues es claro que no solo presentan problemas de adicciones y consumo sino que es relevante que a ellos les afecta de cierto modo la exclusión que generan hacia ellos la sociedad, debido a que como son seres humanos con un sentir, y al evidenciar que los excluye y les son apáticos ocasiona malestar, afectando sus emociones, sumándole a ello la tristeza que les genera el sentirse solos, abandonados y despreciados, generando en ellos un comportamiento agresivo hacia su entorno.

Esta problemática se vivencia en las calles de la ciudad de Bogotá principalmente hacia el centro, puesto que la mayoría de personas que suelen tomar esta decisión bien sea porque les tocó o en muchas ocasiones quisieron, se refugian en donde habitan la mayoría de esta población, para sentirse en compañía de los demás y donde saben que todos están viviendo lo mismo y comparten el habitar en calle.

Las personas que presentan este problema como se ha podido identificar anteriormente son las personas conocidas como habitantes de calle, también percibidas como personas consumidoras y adictas a las drogas, las cuales descuidan su apariencia física y no se preocupan aparentemente de lo que puedan pensar de ellas, sin embargo este pensamiento puede ser subjetivo, debido a que ellos son seres humanos y por ende tienen un sentir como personas, tienen que vivir con la indiferencia de la sociedad y con varios desplantes e incluso se mira a estas personas como un problemas para la sociedad, ya que genera de cierto modo inseguridad, debido al concepto hipotético que se tiene de los habitantes de calle. 
Debido a esto es importante prestarles una buena atención a esta población y en vez de ser intolerantes y mostrarse con repulsión, se debe brindar una ayuda y aportar soluciones que sean viables y óptimas pues esta población carece de infinidad de cosas que conllevan a generar un inmenso malestar, el cual origina afecciones a nivel general en este, incluyendo su salud y en especial su salud mental, por ende con esta investigación se desea buscar las diferentes problemáticas que llevan al habitante de calle a no tener una salud mental adecuada, como: problemas familiares, problemas con la personalidad, pérdida de vínculos familiares y sociales, drogadicción, etc.

En Colombia en el censo del DANE (2020) da un total de 17.960 habitantes de calle, teniendo como mayor relevancia las adicciones a sustancias psicoactivas, evidenciando la Política Pública Social Para Habitante de Calle (PPSHC) para el 2019 son escasas las estadísticas encontradas de habitante de calle por lo cual se pide al Ministerio de Salud y Protección Social (MSPS) un consolidado de cinco ciudades del país de 20092016 con un total de 34.417 habitantes de calle en el que se observa que la tasa más alta de habitantes de calle se encuentra en la ciudad de Bogotá siendo el 50\% de la población total quienes están en la ciudad. De acuerdo a lo anterior se evidencia que en 2020 también se realiza una PPSHC la cual incluye los años 2020-2030, con los censos de 2017-2019 se encuentra en Bogotá y otras 5 ciudades un total de 22.790 habitantes de calle y sigue siendo Bogotá la que mayor concentración de la población tiene con un 41,9\%.

Según las políticas públicas de 2018 y 2020, se evidencia que quieren generar una reducción en la habitabilidad de calle, lo cual muestra que las cifras del 2018 al 2020 se redujeron considerablemente, esto quiere decir que el estado ya está generando un trabajo en conjunto con profesionales para la inclusión y el bienestar social de esta población prestando servicios de desintoxicación, formación académica, hogares de paso y también el trabajo con las familias de los habitantes de calle, ya que son una ficha clave para que ellos vuelvan a tener participación en la sociedad y de esta misma manera generan una prevención a la habitabilidad en calle.

Lo anterior se encuentra en las políticas públicas, donde se observa que en ellas no se estudia ni se pone en práctica el tema de salud mental como un tema relevante en el habitante de calle, ya que dan más importancia al consumo de sustancias psicoactivas y al trabajo de desintoxicación, rehabilitación y la inclusión social, pero no se evidencia el trabajo de los profesionales con las personas habitantes de calle que ya han sido rehabilitados en el tema de salud mental y bienestar. Por ende, se evidencia que para estas entidades no es tan importante este tema, si no que ven más importante la realización de la desintoxicación del consumo de sustancias psicoactivas en la persona que ha vivido un largo episodio de este consumo, lo hace evidente la escasez de estudios en Colombia sobre las personas habitantes de calle.

La búsqueda de información se realiza por medio de bases de datos en línea (Scopus), bases de datos de libre acceso (Scielo, Redalyc, Google Académico). Se incluyen los siguientes descriptores: salud mental, inclusión social, bienestar social, habitante de calle, homeless. Por lo anterior se evidencia que respecto al título del trabajo de grado no se encuentra ningún estudio que abarque el tema completo del trabajo de grado, por lo cual se realiza una búsqueda con los descriptores anteriormente nombrados, con lo cual 
se encuentran artículos de 1985 a 2009 en donde 7 son de estudios internacionales en los cuales se encontraron planes de desarrollo para los habitantes de calle, por parte del Congreso de la República, la Alcaldía Mayor de Bogotá y se evidencia que se maneja a nivel local y de cada país y maneja cada país sus planes.

Con lo expuesto, se desea dar respuesta a la pregunta ¿de qué manera la inclusión social influye en la salud mental y en el bienestar de las personas adultas habitantes de calle? El objetivo central es analizar la manera en que la inclusión social influye en la salud mental y en el bienestar social de las personas adultas habitantes de calle, partiendo de una revisión documental que favorezca la atención integral a nivel psicosocial.

\section{DESARROLLO ACERCA DEL HABITANTE Y LA HABITABILIDAD EN CALLE}

Las definiciones de habitante de calle son variadas, históricas y tienen significados políticos, sin embargo la habitabilidad en calle es una figura social latente en varias culturas, países y en varias ocasiones históricas, por ende en el siglo XXI no hay un acuerdo general de definición exacta, ya que, al no tener ese acuerdo general acerca de una definición precisa se logra deducir que esta población muchas veces no es tomada en cuenta para realizar investigaciones, programas, y políticas que tienen una intervención (Nieto y Koller, 2015).

La definición para habitante de calle dentro de la literatura científica es muy variable debido a que existen varias demarcaciones a esta población, sin embargo, es llamada dentro de una misma lengua en inglés son denominados como; "homeless, shelters, roofless, marginals, y a los menores de 18 años se les denomina Street children, runaway o thrownaway" (Nieto y Koller, 2015, p. 2164). Pero en español son llamados como habitantes de calle, sin techo e indigentes, por lo cual en los censos acostumbran a clasificarlo como personas sin techo. Entonces existen distintas expresiones en una misma lengua y transformaciones en la traducción de un idioma a otro (Nieto y Koller, 2015).

El concepto de habitante de calle, hace referencia a las personas que habitan en la calle, los cuales no tienen hogar y se encuentran relacionados con temas de drogadicción, alcoholismo y exclusión social; sin embargo, en Colombia son conocidos como: gamines, indigentes, vagabundos o desechables. Son llamados habitantes de calle para conceder un reconocimiento social y ser identificados como ciudadanos. (Ceballos, 2018).

Las categorías de Burke que retoman Nieto y Koller, (2015), están compuestas por dos índices de habitabilidad en calle; índices que se dividen por cuatro categorías, las cuales son: la habitabilidad de calle absoluta, hace referencia a todas aquellas personas que viven en la calle, en los puentes o en baldíos abandonados. La habitabilidad en calle relativa se divide en tres rangos; los cuales son: el primer rango son las personas, las cuales son desplazadas en distintas formas, por ejemplo: refugios, casas de huéspedes y casas de amigos. El segundo rango incluye a las personas que tienen que vivir constantemente en una habitación para una persona y por último, el tercer rango incluye a las personas que viven en una casa, pero no tienen un hogar; ósea que en la casa les brindan condiciones básicas de seguridad y el cubrimiento de ciertas necesidades propias de una vivienda, pero 
no un hogar como tal, ya que en un hogar es seguro para vivir, es un lugar cómodo y cálido, teniendo un lugar de identidad y pertenencia. (Nieto y Koller, pag. 2164)

Por lo tanto, en Bogotá, Colombia desde 1997, realizaron cinco censos, en la población de habitante de calle; donde revisaron y estudiaron las definiciones las cuales utilizaron en los primeros cuatro censos de esta población en la ciudad, sin embargo, en el primer censo, el cual fue realizado en la capital en este mismo año, en el cual a esta población se les describió como "indigentes", pero a la vez, se definieron como "personas que han hecho de la calle su Hábitat". Por consiguiente, realizaron el segundo censo a los dos años después en la misma población en Bogotá y allí se les definió como "habitantes de calle" (Nieto y Koller, 2015 p. 2163).

De acuerdo con lo anterior Nieto y Koller, (2015) afirman que no se explicó, cuál era la definición a la que hace referencia dicha población. En el tercer censo de habitantes de calle de la Capital (Bogotá) que se realizó en el 2001, en la nueva denominación de habitante calle, fue "cualquier persona que, voluntariamente o no, decide hacer de la calle su hábitat". Más adelante en el cuarto censo realizado en 2003, Nieto y Koller (2015), p.2170, señalan que existen habitantes "de" la calle y habitantes "en" calle. De acuerdo con esta diferencia, habitante de calle hace referencia a "una persona de cualquier edad que, habitualmente se ha distanciado y ha roto todo vínculo con su familia y hace de la calle su ámbito de vida inmutable”. (p. 2163).

Los términos empleados al definir la población en posición de habitante de calle, en los correspondientes censos realizados en Bogotá, llegaron a sugerir ideas valiosas, pero de una u otra forma eran definiciones vagas, ya que dichas definiciones se balanceaban entre una situación social, como lo es el residir en la calle y hacer de su calle un hogar o un hábitat. Por ende, estas particulares definiciones no fueron lo suficientemente explicadas, ya que no señalaban perspectivas precisas y objetivos tanto de inclusión y exclusión de las personas a ser censadas. Estos autores, para el quinto Censo de Habitantes de Calle de Bogotá, toman la decisión nuevamente realizar, el uso del término habitante de calle, debido a que el término ya estaba lo suficientemente asociado con la población que lo representa (Nieto y Koller, 2015)

Por otro lado, la definición de habitante en calle es el residir en una vivienda a la cual pertenece. Sin embargo, incluyen a las personas que viven en la calle, a quienes lo hacen en sitios especiales de alojamiento para suspender la habitabilidad en calle, y a quienes residen en construcciones como cambuches, carruajes y automóviles. Sin embargo, Nieto y Koller, (2015) proponen la siguiente definición "Toda persona viviendo en la ciudad y que no reside en una vivienda prototípica (casa, apartamento o cuarto) de manera permanente (al menos 30 días continuos) y estable (al menos 60 días en la misma unidad de vivienda) en un momento dado; así como toda persona que reside en un lugar especial de alojamiento como recurso o estrategia para evitar, suspender o terminar con la residencia en viviendas no prototípicas o en calle." (p. 2170). De acuerdo los términos empleados en las discusiones sobre la habitabilidad en calle de los países desarrollados, se puede afirmar que se trata de una definición reactiva, reducida a la dimensión habitacional, operativa y funcional para los estudios cuantitativos y de enumeración, pero que 
relativamente es asociada a la dimensión social de la anterior problemática (Nieto y Koller, 2015).

El siguiente aspecto trata acerca de otra mirada a lo que es habitante de calle y en calle, de modo que, el habitante en calle siempre ha existido y también va a existir y es necesario considerar sus derechos y constricciones, para emplear una mayor conformidad en la convivencia urbana. A partir de la Constitución Política de Colombia, Ruiz (2016) intenta dar una dirección adecuada, para moderar la situación de los habitantes en calle, ya que es una población con gran vulnerabilidad y exclusión en el contexto que se encuentran; por consiguiente, imposibilita encaminar medidas restrictivas contra dicha población. "La dignidad humana y libre determinación de la persona se construye como soportes fundamentales de la Constitución Política de 1991, dependiendo de su atención podría darse una solución para el habitante de calle. (Ruiz, 2016).

Hay poca información y estudios que hablan con respecto a la habitabilidad en calle en adultos mayores, debido a que en su mayoría de estudios e investigaciones son realizados a la población juvenil que habita en la calle, correspondiente a que son muy pocos los adultos y ancianos que lleguen a habitar la calle, ya que si son adultos los que habiten allí, llegaron a las calles cuando eran jóvenes, siendo estos víctimas de consumo excesivo de sustancias psicoactivas. Sin embargo, también presentaron afectaciones en su infancia presentando violencia sexual, afectiva, emocional y física; adicional el no presentarse buenas oportunidades en su vida y bajos ingresos. (Nieto, 2011).

Así que el habitante en calle, es entendido como aquella persona que encuentra un sustento y forma vida en la calle, ya que no es lo mismo el habitante de calle, porque es la persona que, además de lo anterior hace de la calle su hábitat, de modo que para el habitante en calle sería bueno delimitar concertaciones de cohabitación; para el habitante de calle se debe emplear estrategias, las cuales deberán dar soluciones que permitan esquivar que dicha población duerma y enferme en calle, pues esta condición hace más vulnerable a esta población y genera un gran rechazo, riesgo para los habitantes de la ciudad ( Ruiz, 2016).

Según Calaça y Risso (2013) retoman a Marx con el "mendigo" de la modernidad temprana con dos procesos: expropiación y legislación; por lo anterior el primer proceso habla de los ex campesinos que habían perdido sus tierras quienes emigraron a las grandes ciudades y siguiendo el segundo proceso era en el que debían seguir las leyes en la nueva sociedad en la que se encontraban, estas leyes determinaban quien y cuando podía trabajar o ser vagabundo. Lo anterior se evidencia en la actualidad con los diferentes manejos que tienen los gobiernos con la población habitante de calle para que tengan más oportunidades y de esta manera llegar a una vida mejor y no sean excluidos por el resto de la población, con diferentes programas para llegar a su inclusión.

De acuerdo con Gómez (2012) los habitantes de calle que para este año se calculaban 9.600 muchos de ellos tienen paranoia, episodios de esquizofrenia y delirio de persecución, lo que también se une con el mal olor que tienen ellos y el consumo de basuco una de las drogas más económicas que encuentran en el mercado y que es completamente dañina. Se evidencia que la mayoría de los habitantes de calle que tienen enfermedades mentales son consumidores crónicos de basuco. 
Lo anterior da cuenta que si el habitante de calle lleva muchos años consumiendo llega a tener un deterioro en el cerebro y se da inicio a los diferentes problemas de salud mental como los nombrados anteriormente además que les genera un deterioro físico muy evidente; muchos de los habitantes de calle que viven en la ciudad de Bogotá tienen algún estudio no todos son pobres pero llegan a ver en la calle su hogar y también se evidencian problemas en sus hogares lo cual los lleva a vivir en calle desde muy niños. (Gómez, 2012).

En la sociedad colombiana postulan dar el mismo trato al habitante de calle y al habitante en calle, ya que son asociados con el consumo de sustancias psicoactivas, puesto que son dos grupos con gran diferencia y un ejemplo de ellos es el relato de un reciclador en Armenia, quien dijo: "Soy reciclador, no un habitante de la calle. Tengo mi hogar" (Ruiz, 2016).

De lo anterior se puede decir que la población Colombiana, no tiene muy claro que hay dos tipos de habitantes de calle y que de una u otra forma son seres humanos que tienen los mismos derechos como los otros y que por malas decisiones que tomaron en su pasado y malas conductas que emplean a menudo, pero no es porque ellos quieran, es porque la misma población los obliga a ser agresivos, ya que se ha evidenciado tanta discriminación, donde solo un acto de amabilidad no discriminatoria podría cambiar su conducta tan agresiva y se pueda llegar a suprimir la condición de habitante de calle.

Se denota grandemente la afectación a nivel mental que presenta esta población ya que presentan gran vulnerabilidad, son muchos los factores que afectan la salud mental en ellos, al hablar de salud mental se entiende que es un estado de bienestar, es decir estar bien y claro está mentalmente (psicológicamente) es no presentar ningún tipo de perturbación que afecte la salud y se evidencia que diariamente esta población presenta grandes afectaciones.

La vulnerabilidad incluye al sentido de inconsistencia en donde tiene un campo alto de aplicación como: el derecho, la bioética, salud ambiental, epidemiología y salud mental. El concepto de vulnerabilidad social, parte de la relación entre individuo y colectividad, la cual es construida por las relaciones de poder, donde puede referirse a la disminución de derechos sociales y civiles. Por ende, las personas vulnerables tienen poca capacidad al actuar y defenderse, sin embargo, existen entidades las cuales garantizan los derechos fundamentales, tomando los principios de igualdad y de no discriminación; apoyándose en la calidad humana de toda persona. Muchos grupos poblacionales, son más vulnerables que otros, sin embargo, estos grupos distribuyen competencias relacionadas con su posición social, económica, apoyo social y condiciones de vida, donde se incluye el afrontamiento de discriminación en que presentan vivencias de situaciones violentas y de abuso donde tienen una entrada pequeña a los servicios de salud, educación y exclusiones de conveniencias en cuanto a trabajo y beneficios. En cuanto a la salud mental, es primordial distinguir la vulnerabilidad individual, social y funcional de las personas que padecen trastornos mentales y personas que se encuentran en estado de consumo de sustancias psicoactiva, puesto que, la vulnerabilidad puede dar paso a la disminución de salud mental, debido a que esta población, debe afrontarse a la discriminación, lo cual genera baja autoestima, disminución de la autoconfianza, desmotivación con menos sentido de vida. (Arena 2017, pág. 175) 
Los derechos humanos decretan la protección y promoción de los mismos derechos, integrándose a las políticas para una mejor calidad de vida en estas poblaciones, como objetivo de estimular la concientización y autonomía dando paso a una construcción de experiencias positivas, por lo tanto no hay bienes de autonomía, sin hacer conciencia de la condición en la cual se presenta esta población, ya que puede ser un mecanismo para que la persona obtenga un aprendizaje de su propia vulnerabilidad, apropiándose de su realidad con motivo de generar una transformación. Para concluir la población en vulnerabilidad no se puede mezclar con incapacidad, ya que los grupos vulnerables no pueden ser considerados como víctimas de su propio destino (Arena, 2017)

Por consiguiente, la salud mental se debe considerar de atención pública y primordial en la cual el modelo de atención, debe ser óptimo y de accesibilidad para los habitantes de calle, el cual cumpla con las condiciones necesarias y de satisfacción para esta población, para de esta manera evitar el crecimiento de habitabilidad en calle y afectaciones en su salud mental. El sistema de atención integral debe dar cumplimiento frente a lo que plantea la organización mundial de salud (OMS) en cuanto a la "promoción y prevención de posibles apariciones de enfermedades que afectan la salud mental y de esta manera prestar una atención prioritaria para que se pueda llevar un control frente a estas afectaciones a nivel mental, creando en los ciudadanos una perspectiva diferente en cuanto al pensamiento y la importancia que se le da a la salud mental y de tal modo estar alerta frente a los factores psicosociales y conductuales que generan dichas afectaciones en la salud mental y atente contra el desarrollo de la población.”(Urrego y Cocoma, 1999, p. 64)

Según Parales (2008) "La salud mental es un estado de bienestar definido de acuerdo con estándares socioculturales; la tendencia es hacia la consideración de la salud mental en términos positivos. De igual manera nombra la definición que la Organización Mundial de la Salud (OMS) tiene referente a la salud mental la cual define como; un estado de bienestar en el que el individuo desarrolla sus capacidades, afronta las tensiones normales de la vida, puede trabajar productiva y fructíferamente y es capaz de contribuir a su comunidad" (p.663).

Las mujeres habitantes de calle son las que prevalecen en presentar alguna dificultad a nivel de su salud mental, no dejando atrás al género masculino. Los habitantes de calle presentan en su mayoría trastornos mentales graves, evidenciando que este es uno de los factores que conllevan a la persona a habitar en la calle, sin descartar que muchos de estos trastornos también se van generando al pasar del tiempo, en su nuevo hábitat y asociados con tanto consumo de drogas y sustancias que están en la adquisición de esta población.

Según estudios e investigación determinaron que esta población habitante de calle en la cual consideraron que presentan disfunción cognitiva, adicional que presentan cierto tipo de trastornos presentados en su niñez como abusos no solo sexuales, sino emocional, físico conllevando esto a presentar trastornos de personalidad y neuro comportamentales, identificando que esto no tenía consigo ningún tipo de asociación con la presencia de consumo de sustancias. 
Astorquiza (2017) da a conocer las diversas problemáticas internas que presenta un habitante de calle para tomar la decisión de habitar en ella, al igual que los diferentes problemas de salud mental que se evidencian en el habitante de calle como pueden ser, "el trastorno antisocial de la personalidad, el trastorno afectivo bipolar, los rasgos suicidas y los trastornos psiquiátricos como: la depresión y la ansiedad.” (p.36)

Los trastornos de personalidad son de cierto modo los que presentan con mayor frecuencia los habitantes de calle; como lo son el antisocial, histriónico y narcisista, probando que el trastorno antisocial es el de mayor prevalencia debido a que las personas con habitabilidad de calle, al llegar a ese nuevo mundo, lo primero que hacen es aislarse y volverse una persona antisocial, debido a que creen que los van a juzgar, que de cierto modo no los van aceptar, sumándole a esto que se sienten bastante solos, sin querer tener nada que ver con la sociedad y viviendo su vida a su gusto.

Las personas que habitan en calle presentan graves problemas mentales y no solo asociados con consumo de sustancias psicoactivas sino también las condiciones en las que viven ya que es claro que no es la mejor y esto de cierto modo genera incomodidad ocasionando afectaciones mentales, puesto a que son personas a las cuales excluyen bastante y presentan vulnerabilidad, el estar en calle, solo, aislado, sin poder satisfacer sus necesidades origina grandes problemas en su salud mental. Sumándole a esto que son una población a la cual no se le presta el más mínimo apoyo con respecto al acceso de salud y menos al de la salud mental, esto causa afectaciones de tipo emocional ya que no cuentan con apoyo esencial.

Cuando el ser humano presenta afectaciones en su salud mental se evidencia indudablemente puesto a que no se puede desenvolver de la misma manera y también comienza a presentar y generar más afectaciones a nivel emocional, debido a que se presentan diferentes cambios, el habitante de calle puede presentar episodios de depresión, ansiedad, estrés, se recrean traumas y enfermedades mentales debido a esto es bueno reconocer que ese sentir no es bueno y se tiene el derecho a buscar ayuda.

En cuanto a la ley 1641 de 2013 se encontró información en el artículo de Bernal y Londoño (2016), donde mencionan el objetivo principal de la ley el cual es escrito textualmente "establecer los lineamientos generales para la formulación de la política pública social para habitantes de la calle dirigidos a garantizar, promocionar, proteger y restablecer los derechos de estas personas, con el propósito de lograr su atención integral, rehabilitación e inclusión social.” (p.761).

Mencionan la ley 1641 de 2013 la cual indican que es la que intenta combatir la situación de la habitabilidad en la calle a través del establecimiento de los derechos humanos, se habla también de la diferencia que hay entre indigencia y habitabilidad en calle ya que por el simple hecho de que son poblaciones vulneradas y rodeadas de pobreza las suelen confundir y asociar.

En la ley anteriormente nombrada, definen al habitante de calle textualmente como; "persona sin distinción de sexo, raza o edad, que hace de la calle su lugar de habitación, ya sea de forma permanente transitoria y, que ha roto vínculos con su entorno familiar" (Gómez, 2013, p.29). 
Los derechos los cuales tiene esta población por el simple hecho de su existencia como ser humano y hacer parte de la ciudadanía, las personas habitantes de calle son señaladas y discriminadas por la sociedad llevando a cabo una exclusión social con esta población la cual también hace parte de la ciudadanía, en esta población se puede encontrar desde bebes hasta adultos mayores, suelen refugiarse en lugar muy alejados y si es posible que no sean visibles.

Para continuar hablando de problemas de salud mental en habitantes de calle se encuentra un estudio realizado por Gowda, Chithra, Moirangthem, Kumar, Math en el año 2020 en India con todo lo que ha conllevado la pandemia por el COVID 19 y que además tienen algún problema de salud mental. Gowda, Chithra, Moirangthem, Kumar, Math, (2020) realizan un trabajo con la protección de esta población, dando a conocer las diferentes formas de arduo trabajo con la organización de las naciones unidas y entidades no gubernamentales y de cuidado que en India se debe tener para las personas sin hogar. Para los tiempos de pandemia se les requiere la asignación de un lugar en el cual esta población tenga en donde resguardarse y en ese mismo lugar tener todas las medidas de bioseguridad al igual que personas que los acompañen y les den orientación sobre cómo es el lavado de manos, uso de antibacterial, etc. Con lo anterior se evidencia que esta población llega a tener episodios de abstinencia y de diferentes problemas de salud mental en pandemia ya que se genera un toque de queda en toda India para que la propagación del virus no sea mayor por lo cual se genera una necesidad de tener servicios psicosociales para la población Habitante de calle (HC) para abordar los problemas de salud mental que se generan en la población.

Frente a la población que es más vulnerable al contagio de este virus se debe tener aún mucha más comprensión, ya que es importante transmitirles tranquilidad que evidencia un apoyo emocional por parte de sus familiares, cuidadores y profesionales claramente se les debe hablar con suavidad, claridad, de manera respetuosa adicional priorizándolos a ellos y evitando cualquier tipo de contacto con el virus, en cuanto a los niños es bueno mantenerlos distraídos que jueguen, realicen actividades como cantar, bailar, incluir actividades de aprendizaje, es importante que se ayude a que los niños sepan cómo expresar sus emociones y de la misma manera cómo manejarlas, estar siempre bajo el cuidado de los padres es fundamental evitar cualquier afectación psicológica en ellos. (Organización mundial de la salud, 2020)

Se desea determinar cuáles son las necesidades de las personas habitantes de calle que presentan problemas en su salud mental y muestran signos de consumo excesivo de sustancias psicoactivas y consumo de bebidas embriagantes, conociendo que prima el género masculino en esta población que se encuentran en situación de calle. (Grandón, Vielma, Castro, et. al, 2018).

A la población de personas sin hogar no se les atiende de manera muy buena en las diferentes entidades de salud por lo cual se vuelve un desafío encontrar un psicólogo o psiquiatra que tenga acompañamiento directo con la población, ya que por la pandemia muchos de ellos trabajan por tele orientación y en la situación en que se encuentran las personas sin hogar es muy complicado tener acceso a un teléfono o un celular con algún tipo de conexión a red de internet para de esta manera poder tomar la cita, por lo cual se 
evidencia que para una pandemia como la de COVID 19 es muy difícil el trabajo la salud mental con el habitante de calle y están más expuestos a sufrir de patologías que podrían tener por el encierro y por el estrés que les genera todo el tema de un contagio en la condición en la que se encuentran y más que todo si son población priorizada.

En Colombia se encuentra la Guía Metodológica para el Observatorio Nacional de Salud Mental lo cual se evidencia que mental es una instancia técnica que se responsabiliza de consolidar y dar información sobre salud mental de la población colombiana, así como también a los diferentes servicios políticos de cada territorio y programas que trabajan con los diferentes trastornos mentales, se evidencia que se apoyan las líneas de investigación en salud, observar cómo trabajan los diferentes lugares que tienen atención a salud mental, tienen estrategias para que se mejore el estado de salud mental de Colombia. El observatorio nacional de salud mental también trabaja realizando la divulgación de los diferentes modelos y programas de atención en salud mental para que de esta manera sea más óptimo, al igual que mejorar el trabajo con la población colombiana y la prevención de la salud mental en la población. (Grupo Funcional: Gestión Integrada para la Salud Mental, 2017)

La población con habitabilidad en calle ha aumentado en grandes cantidades ya que las personas que toman la decisión de comenzar una nueva vida en las calles no solo son las personas que consumen drogas sino también personas que carecen considerablemente de oportunidades en el país y en la ciudad, son personas que en su mayoría efectivamente llegan a situación de calle debido al consumo de sustancias psicoactivas y bebidas alcohólicas, pero el resto de esta población también están allí por falta de recursos, por desempleo, desplazamiento, problemas económicos, falta de educación y violencia intrafamiliar. (Otalvaro y Correa, 2009).

Por lo anterior en el lineamiento para atención diferencial en salud para población habitante de calle en Colombia, se encuentra que existe muy poca oferta en los servicios de salud mental al igual que existen escasos funcionarios que tengan conocimiento para trabajar con la población y también en salud mental por lo cual no se encuentran servicios de atención para la población. Es necesario que para la atención de salud mental en la población se logren diagnosticar a tiempo para iniciar tratamiento con una ruta específica para ello. (Grupo Gestión Integral de la Promoción Social, 2019).

La salud mental se debe considerar inmediatamente de atención pública en la cual el modelo de atención, tiene que ser prestado para la atención a los habitantes de calle se debe diseñar e implementar un modelo de salud mental que cumpla con las condiciones para esta población y evitar el crecimiento. (Castro, 2017).

La salud mental es fundamental y se le debe prestar una atención oportuna, por eso el video de Cielo de lo bueno y útil que es formarnos y conocer más a fondo lo importante que es tener una buena salud mental de la cual nos debemos preocupar y no avergonzarnos, es por eso que se debe educar y desde pequeños enfatizar en que al ser humanos se tiene un sentir y que hay cosas buenas y malas en todos los sentidos y se debe expresar libremente sin miedo alguno todo lo que se sienta. (Cielo, 2019). 
Según Borysow y Furtado, (2013) la acogida y garantía de los problemas de salud mental como objetivo tiene la utilización de red de servicios descentralizados y territorializados como lo son Centros de Atención Psicosocial, otra para el tratamiento de uso abusivo de alcohol y otras drogas, para la atención los Servicios Residenciales Terapéuticos y los Centros de Convivencia, configurando el nuevo modelo de atención en salud mental en el Brasil. Las unidades básicas de salud en Brasil para las personas en situación de calle las cuales han considerado como puerta de entrada para los problemas de salud mental graves en esta población; por lo anterior las personas en situación de calle no tienen un mayor acceso a los servicios de salud mental por diferentes políticas públicas relacionadas con la dificultad de organizar acciones en calle y la complejidad que se tiene al incluir esta población en dichos programas.

Frente al acceso de los servicios de salud mental se presentan varias dificultades para poder acceder al mismo empezando por las afectaciones económicas, geográficas y culturales, generando una gran serie de limitaciones, viendo un deterioro significativo frente a la atención en la salud mental fomentando enfermedades graves y no llevando a cabo la prevención de las mismas. (Rojas, Castaño y Restrepo, 2018).

La pirámide de servicios de la salud mental elaborada por la OMS creando una combinación óptima dividida en 5 cantidades de servicio ubicándose 2 en el cuarta parte de abajo hacia arriba, iniciando por la auto atención, siguiendo la atención comunitaria informal, posteriormente los servicios de salud mental a través de la atención primaria de la salud, en el cuarto dividiéndose en dos los cuales son servicios de salud mental comunitarios y servicios psiquiátricos en hospitales generales y finalizando en las instalaciones para estancias de larga duración y servicios especiales. (Ferro, 2010).

Para empezar a hablar de inclusión social en las personas habitantes de calle parte en enunciar que existe un problema en el gobierno en América Latina robustecido por la economía mundial lo cual da cuenta que en Latinoamérica existen 3 características importantes que son: el patrimonialismo, el autoritarismo y la exclusión lo anterior mostrando que la exclusión se refiere a problemas de pobreza y desigualdad. (Garavito, 2017).

De acuerdo con Garavito, (2017) hace referencia a procesos de ámbito sociopolítico y económico; las personas están en el mercado ya sea como vendedoras o como consumidoras, en la inclusión lo que respecta a la política dice que cada personas tiene derechos y deberes en cualquier comunidad en la que habiten, lo anterior garantiza acceso a bienes públicos, educación y a participación como ciudadanos, lo cual deja ver que la inclusión social es la pretensión por medio de las políticas públicas de deliberación especialmente las políticas públicas sociales.

Por otro lado, el decreto 897 de 2015 no da lineamientos específicos para el cuidado de los HC, pero si se encuentran acciones para realizar en torno a la inclusión social del habitante de calle, en el decreto también se habla que los habitantes de calle pueden llegar a participar como ciudadanos en las decisiones de gobierno, lo cual, en la alcaldía de Gustavo Petro en Bogotá, hace que se haga más importante realizar derechos de las personas habitantes de calle. Se pone en marcha el "Proyecto 7312: Atención integral para la inclusión social de ciudadanos y ciudadanas habitantes de la calle” (Garavito, 2017. 
p.14). En el proyecto se realiza un gran énfasis en la acogida de las personas habitantes de calle incluyendo los hogares de paso que tenían tres jornadas; en el día, en la noche y díanoche y cada hogar de paso tenía servicios; aseo personal, atención en salud y alimentación, lo anterior dándole una gran importancia a la inclusión social del habitante de calle visto desde los hogares de paso en Bogotá con dos alcaldías la cuales también trabajaron en decretos para los derechos de la población.

Sin embargo la biblioteca pública es un lugar que presta servicios e instalaciones, a las personas que no tienen un hogar, la cual brinda un servicio igualitario, no obstante esto quiere decir que no importa la raza, edad, sexo, religión, nacionalidad o condición social, por lo tanto el articulo tiene como objetivo mostrar la colaboración de la biblioteca en función social, cultural y educativa, ya que puede tener una trasformación social en su entorno, estableciendo vínculos con demás entidades, con el fin de buscar un aprovechamiento mutuo; tanto de recursos como fortalezas para poder desarrollar acciones empleando estrategias para mejorar las condiciones y llegar a la inclusión social en habitantes de calle. La biblioteca pública promueve las oportunidades equivalentes en sus usuarios, en cuanto a la información, creación y funcionamiento de las estrategias, las cuales deben permitir la satisfacción de las necesidades de la población a la que atiende para lograr una interacción social. (Ceballos, 2018).

Por lo tanto, Ceballos toma como referencia la Unión Europea, quien define la inclusión social, en la revista Humanum como el "proceso que asegura que aquellos en riesgo de pobreza y exclusión social tengan las oportunidades y recursos necesarios para participar completamente en la vida económica, social y cultural disfrutando un nivel de vida y bienestar que se considere normal en la sociedad en la que ellos viven" (Ceballos, 2018.p.65).

La biblioteca establece las siguientes estrategias para laborar con personas adultas habitantes de calle: “a) Creación de programas de alfabetización para los habitantes de calle, b) Implementación de servicios de información social sobre derechos y oportunidades,

c)Hacer uso del Servicio de Información Local (SIL) para conectar a los usuarios con diferentes centros sociales y organizaciones involucradas en el mejoramiento de la calidad de vida de esta población, d)Aprovechamiento de las diferentes herramientas Web 2.0 para buscar miembros de la familia y vincularlos de nuevo con ellos, e)Creación de programas de lectura y escritura, f)Recopilación de historias y jerga callejera, mediante la realización de talleres literarios, g) Visita a los diferentes lugares de refugio y rehabilitación para promover la lectura y demás servicios bibliotecarios h) Realización de campañas de solidaridad dentro de la biblioteca, por ejemplo, la recolección de vestuario y alimentos para donarlos a los diferentes centros para habitantes de calle, i)Formación de los demás usuarios para concientizarlos sobre las concepciones erróneas y desestigmatización de los habitantes de calle, j) Creación de alianzas estratégicas con diferentes instituciones, con el propósito de unir esfuerzos para desarrollar acciones dirigidas a mejorar la calidad de vida de este sector social, k)Formación del personal bibliotecario para garantizar una adecuada intervención de los habitantes de calle, l) Creación de pequeñas bibliotecas satélites en los centros especializados para los habitantes de calle, m) Realización de talleres de 
sensibilización dentro y fuera de la biblioteca, con temas como la drogadicción, el alcoholismo, la violencia familiar, entre otros, n) realización de charlas motivacionales." (Ceballos, 2018. P.13).

Lo anterior es importante debido a que por medio de estas estrategias, se puede lograr una gran proyección para la realización de la formalización en el habitante de calle, ya que es un tema complejo de trabajar, porque una persona que ha permanecido tanto tiempo en calle, al ver que una entidad lo acoge no se va sentir a gusto y es un poco probable que pueda generar una entidad, hay que tener en cuenta que para que una entidad tome la decisión de trabajar con el habitante de calle, la persona tendrá que poseer una voluntad para que después de la desintoxicación no continúe su vida primero en la calle y segundo en el consumo.

Según el Ministerio de Educación de Colombia (2012) la inclusión social es definida como "las acciones que se realizan para lograr que los grupos que han sido social e históricamente excluidos por sus condiciones de desigualdad o vulnerabilidad puedan ejercer su derecho a la participación y sean tenidos en cuenta en las decisiones que les involucran" (Grupo de Gestión Integral en Promoción Social, 2018. p.18), lo anterior evidencia que la inclusión social se desarrolla en las poblaciones que han sido excluidas y vulneradas y que se quiere llegar a tener una inclusión social de las mismas para que tengan derechos como el resto de la población que no es vulnerada como ellos.

La Alcaldía Mayor de Bogotá habla de la inclusión social en los Lineamientos Generales de Política Social Para Bogotá de 2004-2014 entendida como "la capacidad del Estado y de la sociedad para generar las condiciones materiales que permitan a los ciudadanos ser parte de una colectividad y de una organización social que garantiza sus derechos de forma integral”' (Grupo de Gestión Integral en Promoción Social, 2018. p.18) visto de esta manera se puede evidenciar que en Bogotá se quiere trabajar también que los ciudadanos lleguen a estar en una sociedad como los demás con los mismos derechos y que se cumplan en su diario vivir y que no tengan más exclusión.

De acuerdo con Ursin (2016) al referirse a la inclusión social en las personas sin hogar, se evidencia que es cuestión de poder tener acceso a diferentes espacios en los que ellos quieran acceder para de esta manera convivir con los demás, pero públicamente no es de esta manera lo cual hace que no se incluyan en las diferentes actividades habituales para todo ser humano y el acceso a lugares es muy restringido.

Al hablar de bienestar se inicia hablando del estudio de las políticas públicas en Colombia que llega a ser muy importante en el bienestar social ya que después de que la Constitución Política de 1991 se consolida, se decretó que Colombia fuera un estado social de derecho lo cual lleva a que el bienestar es foco del estado garantizando los derechos sociales. Las diferentes políticas públicas se van desarrollando a medida que la comunidad los requiere entendiéndose que es para el mejoramiento de su vida y dando un mejor bienestar. Retomando lo anterior en el país no se da la posibilidad de un bienestar social de la mano con las políticas públicas ya que existe mucha inequidad y exclusión social lo cual genera mucha desigualdad social más evidente en el tema socioeconómico, dando cuenta que Colombia es uno de los países más desiguales del mundo (Mejía, 2012). 
De acuerdo con Moreta, Gabior y Barrera (2017) el bienestar social se define como el valor que se le da a las circunstancias y cómo funciona dentro de una sociedad; se dan a conocer cinco dimensiones las cuales son:1) integración social la cual da a entender la calidad de las relaciones que se tienen con la sociedad y la comunidad, 2) aceptación social que es el sentirse perteneciente a una sociedad y que esta pertenencia a la comunidad se disfrute,3) contribución social confianza hacia los demás y poder ofrecer algo útil al mundo, 4) actualización social entendida como que la sociedad es dinámica lo cual evidencia que va cambiando y cada individuo cambia con ella y de esta manera puede conseguir de una mejor manera sus objetivos y metas propuestos, 5) coherencia social es la preocupación por enterarse que pasa en el mundo.

Teniendo en cuenta los siguientes conceptos de bienestar social, se evidencia que el bienestar social es una forma de darle una mejor calidad de vida a las personas, lo cual visto desde la población habitante de calle es muy complejo ya que ellos por ser una población tan excluida socialmente no se enfatiza en su bienestar, también se evidencia que Colombia es uno de los países más desiguales del mundo por lo cual no para toda la población colombiana hay un claro bienestar social y no se evidencia tampoco con el habitante de calle ya que muchas veces se deja de lado la población.

\section{DISCUSIÓN}

La revisión de literatura evidencia que hay diversas leyes que buscan una mejor calidad de vida para el habitante de calle; las cuales son; Decreto 646 plan económico, social, ambiental y de obras públicas en los años 2012-2016, Acuerdo 366 de 2009 Establecimiento de lineamientos para atención, inclusión y el mejoramiento de calidad de vida del habitante de calle, Ley 1641 de 2013 Establecimiento de algunos lineamientos, para una política pública social para habitantes de calle, Política Pública Social Para el Habitante de Calle de 2018, Resolución 756 del 2017 Reglamento de comité operativo para la población habitante de calle, Decreto 560 de 2015 Alcaldía Mayor de Bogotá orientación, promoción, protección, recuperación y obtención de derechos de los ciudadanos habitantes de calle de Bogotá, Decreto 471 de 2015 autocuidado, y acogida para los habitantes de calle que se encontraban situados en Los Mártires.

Según las leyes nombradas anteriormente se evidencia que en cuanto al decreto 646 no se ha observado el plan económico, social y ambiental para el habitante de calle el cual tenía una caducidad del año 2012 a 2016; sin embargo, se puede interpretar que este plan fue diseñado solo por un requerimiento, pero no se lleva a cabo. En cuanto al Acuerdo 366 de 2009 se evidencia que en los hogares de paso y algunas fundaciones parten de una atención, una inclusión y el mejoramiento de vida de los habitantes de calle. En cuanto a la Ley 1641 de 2013 es muy parecida al anterior acuerdo, pero esta ley se basa más en las diferentes formas y lineamientos de cómo se debe llegar a la inclusión y la atención con el habitante de calle; no obstante, es importante destacar que la política pública menciona algunos aspectos de la salud mental en cuanto al habitante de calle como la definición y algunos porcentajes de patologías evidenciadas en algunos de ellos.

En cuanto a la Resolución 756 de 2017 Reglamento de comité operativo para la población habitante de calle, es evidente que se establece un reglamento para la población habitante de calle tomada del Acuerdo 366 de 2009 se observa que la resolución le da 
fuerza, al acuerdo, pero no es trabajado adecuadamente. El Decreto 560 y 471 de 2015 estos dos decretos van de la mano con la Alcaldía Mayor de Bogotá, debido a que manejan temas de promoción, prevención, autocuidado, acogida y protección en cuanto a los derechos humanos del habitante de calle; por ende se evidencia que existen los hogares de paso donde se vela por el trabajo de autocuidado con la población, los cuales son; cambio de vestimenta, corte de cabello, implementan estrategias para la elaboración de producto hecho por ellos o dado por el hogar para la venta para suplantar las necesidades en el hogar como; elementos de aseo, alimentación y vestimenta.

Se encontró que las diferentes entidades públicas de la ciudad de Bogotá en relación al habitante de calle abarcan el sistema normativo en el cual se beneficie esta población, llevando a cabo una serie de acciones en las cuales hagan sentir al habitante de calle acogido en la sociedad, manifiestan estas entidades querer favorecer las condiciones en las que habita esta población, estableciendo lineamientos en cuanto a el mejoramiento de la calidad de vida de los mismos, donde se les garantice un mejor estilo de vida donde prime su salud, se vela por los derechos que tienen, por pertenecer a la sociedad también y ser humano el cuanto siente, vive, sueña, etc. El cual tomó la decisión de hacer de la calle su hogar.

En relación a la psicología y la salud mental en el habitante de calle, la normatividad manifiesta ser de gran importancia, la salud integral de esta población en cuanto a que se lleve consigo una atención completa la cual garantice a los ciudadanos de esta población su derecho fundamental a la salud y rehabilitación, se desea hacer sentir al habitante de calle como un ser humano con las mismas capacidades de todos los ciudadanos, y de esta manera prevenir que se sientan inferiores a los demás y entiendan que como personas valen también y que deben tener una vida con igualdad de oportunidades, para que de esta manera se fortalezca su desarrollo como persona. Como desventaja se encontró que frente a la atención de la salud mental en esta población es muy mínima, ya que no cuenta con una gran serie de profesionales expertos los cuales le puedan dar acompañamiento es por eso que no se tiene servicios de atención, es importante realizar un diagnóstico a tiempo para poder iniciar un tratamiento con una ruta específica.

Se observa que se han llevado a cabo una serie de programas y planes en los cuales se habla del bienestar social en el habitante de calle en donde se desea mejorar las condiciones de vida y promover el bienestar de esta población mejorando sus condiciones de vida tanto sociales, territoriales, físicas, psicológicas, etc. Se plantea también poder tener intervenciones sociales.

Se encontró que estas entidades en relación con la inclusión social son de gran importancia para hacer esta población parte de la ciudadanía, ya que ellos también son ciudadanos, por lo cual la inclusión social hace que tengan importancia para la sociedad, teniendo en cuenta que son personas con los mismos derechos. Se abrieron hogares de paso en los cuales pueden tener servicio de aseo, higiene, atención en salud y alimentación, mostrando de esta manera que la inclusión social es dar calidad de vida, y mejorar las relaciones que se tiene con los demás. 


\section{CONCLUSIONES}

La inclusión social influye en los habitantes de calle en cuanto a su bienestar y su salud mental, a pesar de que se tiene olvidada de cierta manera esta población y ellos ya se limitan a vivir el día a día sin ningún tipo de complicación en su diario vivir, debido a que de una u otra forma se resignan a ser ciudadanos que habitan en la calle y que por la gran mayoría de ciudadanos son rechazados o más bien no incluidos de la manera adecuada en la sociedad, determinando que si se generan afectaciones en cuanto a su salud mental ya que, al sentirse ellos como personas que no aporten nada a la sociedad y las cuales tomaron la decisión de habitar en la calle, no son personas bien percibidas por los demás y de esta manera se genera una exclusión de la misma y por esto se procede a llevar consigo afectaciones a nivel psicológico.

Es importante mencionar que si se llega a tener una colaboración por parte del habitante de calle se puede evidenciar una mejoría en su calidad de vida, y su participación en los diferentes programas que se manejan para la población, sin embargo se debe tener en cuenta que hay una variación dependiendo de cómo la población evidencia el cambio en sus pares y cómo se maneja el trato con la misma, por parte de las entidades, ya que es muy importante que se desarrolle la empatía en estos campos y también que las entidades cumplan los requisitos que estipula el Congreso de la República en la ley 1641 de 2013,por tanto mencionan algo fundamental y es que las instituciones que presten un servicio a esta población logren reestablecer los derechos, protegiendo los mismos, promocionando y deberán realizar actividades de inclusión social.

Se puede mencionar que los componentes asociados con el bienestar social del habitante de calle están relacionados con la vivienda, educación, alimentación e higiene, los cuales no se llevan a cabo debido a que la vivienda en esta población se puede ver reflejada siempre y cuando el habitante de calle esté vinculado a una entidad que trabaje con la población, ya que normalmente la población está ubicada en baldíos desocupados, debajo de los puentes y en diversas partes de la ciudad; la educación que ellos tienen es de diferentes niveles debido a que muchas de estas personas han estudiado hasta especializaciones pero por diferentes situaciones no llegaron a ejercerlo y terminaron en calle, en cuanto a las condiciones alimenticias se evidencia que son precarias debido a que ellos se alimentan de lo que encuentran en calle, sin embargo esta población tiene derecho a asistir a los comedores comunitarios, hogares de paso y diferentes entidades que les presten el servicio de alimentación, educación, vestimenta, higiene y presentación personal, por lo tanto en la atención integral en salud se maneja tanto la salud física como mental, lo anterior no se evidencia en gran medida ya que los sistemas de salud no son lo más propensos a atender grandes poblaciones y esta es de las que menos atienden.

Sin embargo, es importante mencionar que la atención integral debe implantar estrategias que traten a profundidad tanto la atención física como la atención psicológica en donde se vea reflejado la formación ciudadana con el objetivo de promover entornos saludables y restauración de vínculos afectivos en donde se involucra a la familia.

Para implementar lo anterior se pueden plantear unos indicadores con los cuales se realice seguimiento exhaustivo, por lo cual se encuentran diferentes tipos de estrategias como lo son el impulsar programas para el fortalecimiento de las capacidades, entre ellas; 
la educación y el arte, en pro de generar ingresos y mejorar sus posibilidades de inclusión laboral y de esta manera desarrollar campañas de sensibilización con la sociedad y especialmente con los sectores productivos para que vinculen de manera productiva a los habitantes de calle.

El trabajo de grado se dirigió en cuanto al enfoque humanista debido a que muchas investigaciones hablan de la población y dejan de lado lo personal, en cuanto a la salud mental se buscó información en cuanto al autocuidado y las técnicas que manejaban en cuanto a lo personal, evidenciando pautas de autocuidado con el habitante de calle, estrategias de aprendizaje para una inclusión social y re significación de derechos humanos.

Se encontró una limitación a lo largo de la investigación, debido a que el tema del que se investigó en el trabajo de grado, cuenta con muy poca información, es decir que no hay una gran variedad de indagación sobre el mismo, por el contrario de lo que se puede observar, la mayoría de inquisición que se puede encontrar son investigaciones con el habitante de calle, pero en relación a su habitabilidad allí y en cuanto al consumo de SPA. Sin embargo, se encontró un gran cuadro normativo donde se evidencian decretos, leyes, acuerdos entre otros, mediante los cuales se "cobijan" y velan por los derechos humanos y el bienestar de los habitantes de calle, pero se pudo apreciar que no se cumplen del todo esa serie de normatividades, lo cual da cuenta que no se trabaja con la población como se espera e incluso de cómo se lo merecen por ser ciudadanos colombianos y tener los mismos derechos y deberes que los demás.

\section{REFERENCIAS BIBLIOGRÁFICAS}

Alcalde Mayor de Bogotá (21 de diciembre del 2015). Decreto 560 de 2015. Alcaldía Mayor de Bogotá, D.C. Registraduría Distrital. https://www.alcaldiabogota.gov. $\mathrm{co} /$ sisjur/normas/Norma1.jsp?i=64210\&dt $=\mathrm{S}$

Alcalde Mayor de Bogotá (2016) Decreto 646 de 2016. Secretaria Jurídica Distrital. Recuperado de: https://www.alcaldiabogota.gov.co/sisjur/normas/Norma1. jsp? $\mathrm{i}=67808$

Arena, C. (2017) Salud Mental y Vulnerabilidad: desafíos y potencialidades en la utilización del referencial de los derechos humanos. Electrónica Saúde Mental Álcool Drog. 13(4) 174-175. Recuperado de:

http://pepsic.bvsalud.org/scielo.php?script=sci_arttext\&pid=S1806-697620170004000 01\&lng=en\&nrm=is.\&tlng=es

Astorquiza, J, (2017) Salud mental en el habitante de calle, usuario de la Casa de Paso

Camino de Esperanza, desde un enfoque humanista integral. Boletín Informativo CEI. Recuperado de: http://editorial.umariana.edu.co/revistas/index.php/Boletin InformativoCEI/article/view/1367

Bernal, M \& Londoño, V (2016) Habitantes de calle, ¿ciudadano sujeto de derechos? Una revisión a la política pública nacional para habitante de calle en la ciudad de Bucaramanga. Revista cambios y permanencias: Número 7, 2016, pp.757770. 
Recuperado de https://revistas.uis.edu.co/index.php/revistacyp/article/view/ $7072 / 7315$

Borysow, I y Furtado,J. (2013) Acesso e intersetorialidade: o acompanhamento de pessoas em situação de rua com transtorno mental grave. Physis Revista de Saúde Coletiva, Rio de Janeiro, 23 (1). Recuperado de: https://www.scielo.br/scielo.php?pid= S0103-73312013000100003\&script $=$ sci_abstract\&tlng $=$ pt

Calaça, Q y Risso, W. (2013) Cooperatives of waste pickers as an alternative to social exclusion and its relationship with the homeless population. Faculdade de Saúde Pública, Universidade de São Paulo. DOI 10.1590/S0104-12902014000100011.

Castro, N (2017) Youtube. 17/01/17 Situación Habitante de calle, adicciones y salud mental. Recuperado de: https://www.youtube.com/watch?v=wjBaplUvz0k

Ceballos, J. (2018). El rol de la biblioteca pública en la inclusión social del habitante de calle (homeless). Investigación Bibliotecológica, 32 (76), 63-78 Recuperado de: http://www.scielo.org.mx/scielo.php?script=sci_arttext\&pid=S0187-358X20180 00300063

Cielo, V. (2019) Youtube. La importancia de la SALUD MENTAL. Recuperado de: https://www.youtube.com/watch?v=8X-IYDdi-DA

Congreso de la República (12 de julio del 2013). Ley 1641 de 2013 Diario oficial. Recuperado de: https://www.icbf.gov.co/cargues/avance/docs/ley_1641_ 2013.htm\#1 DANE (2020) Censo Habitantes de la Calle 2019, DANE Recuperado de https://www.

dane.gov.co/files/investigaciones/boletines/censo-habitantes-calle/consolidadocinco ciudades-2019.pdf

Ferro, R. (2010) SALUD MENTAL Y PODER Un abordaje estratégico de las acciones en salud mental en la comunidad. Revista de Salud Pública, (XIV) 2:47-62, dic. 2010: https://revistas.psi.unc.edu.ar/index.php/RSD/article/viewFile/7082/8155

Gómez, C (2013) El habitante de la calle en Colombia: Presentación desde una perspectiva social-preventiva. Actualidad jurídica: https://observatoriodeviolencia. dosquebradas.gov.co/wp-content/uploads/2018/08/El-habitante-de-la-calle-enColombia.pdf

Gómez, Y. (2012,11 de febrero). La mitad de habitantes de la calle en Bogotá tiene problemas mentales. El Tiempo. https://www.eltiempo.com/archivo/ documento/ CMS-11117341

Gowda.G, Chithra. N, Moirangthem. S, Kumar. C, Math. S (2020) Homeless persons with mental illness and COVID pandemic: Collective efforts from India. Asian Journal of Psychiatry 54(20) DOI: https://doi.org/10.1016/j.ajp.2020.102268

Grandón F., Pamela, Vielma-Aguilera, Alexis, Castro-Alzate, Elvis Siprian, Bustos N. Claudio, \& Saldivia B., Sandra. (2018). Caracterización de las personas en situación de calle con problemas de salud mental, que se encuentran en la Región del BíoBío. 
Revista chilena de neuro-psiquiatría, 56(2), 89-99: https://scielo.conicyt.cl/ scielo.php?pid=S0717-92272018000200089\&script=sci_arttext

Grupo Funcional: Gestión Integrada para la Salud Mental. (2017, Enero).Guía Metodológica para el Observatorio Nacional de Salud Mental. Subdirección de Enfermedades No Transmisibles https://www.minsalud.gov.co/sites/rid/Lists/ BibliotecaDigital/RIDE/VS/ED/GCFI/guia-ross-salud-mental.pdf

Grupo Gestión Integral de la Promoción Social. (2019, agosto). Lineamiento para atención diferencial en salud para población habitante de calle. Oficina de Promoción

Social. https://www.minsalud.gov.co/sites/rid/Lists/BibliotecaDigital/RIDE/DE/PS /lineamiento-salud-habitante-de-calle.pdf

Grupo de Gestión Integral en Promoción Social. (2018, Abril). Política Pública Social Para Habitante De Calle - PPSHC-. Oficina de Promoción Social. https://www.minsalud.gov.co/sites/rid/Lists/BibliotecaDigital/RIDE/DE/PS/ politica-publica-social-habitante-de-calle.pdf

Mejía, J. (2012). Modelos de implementación de las políticas públicas en Colombia y su impacto en el bienestar social. Analecta política. 2(3) p.141-164.

Moreta, R. Gabior, I. Barrera, L. (2017). El Bienestar Psicológico y la Satisfacción con la Vida Como Predictores del Bienestar Social en una Muestra de Universitarios Ecuatorianos. Salud \& Sociedad. 8 (2) p.172-184. https://www.redalyc.org/ pdf/4397/439752880005.pdf

Nieto, C \& Koller, S (2015). Definiciones de Habitante de Calle y de Niño, Niña y Adolescente en Situación de Calle: Diferencias y Yuxtaposiciones. Acta de Investigación Psicológica 5 (3) p.2162 - 2181 Recuperado de: http://www.scielo.org.mx/scielo.php?pid=S200748322015000302162\&script=sci _arttext\#B40

Nieto, C (2011) Consumo de drogas en tres etapas de la vida de habitantes de calle de Bogotá: predictores de consumo y comparación con una muestra de población infantil y adolescente de Brasil. Universidad federal do rio grande do sul: https://www.lume.ufrgs.br/bitstream/handle/10183/37297/000820609.pdf?sequ ence $=1$ \&isAllowed $=\mathrm{y}$

Organización mundial de la salud (2020) Consideraciones psicosociales y de salud mental durante el brote de COVID-19.

Parales, C (2008) Anomia social y salud mental pública. Salud Pública. Recuperado de: https://www.scielosp.org/article/rsap/2008.v10n4/658-666/es/

Ruiz.J.P. (20 de septiembre del 2016). Habitante en calle versus habitante de calle. El espectador. https://www.elespectador.com/opinion/opinion/habitante-en-calleversus-habitan-de-calle-1-columna-655842/

Rojas. L, Castaño. G, Restrepo. D. (2018). Salud mental en Colombia. Un análisis crítico. Rev CES Med. 32(2): 129-140. 
Urrego M, Diana Z y Cocoma P, Juan C. (1999). Por la Salud Mental. Revista de Salud Pública, 1 (1), 58-67. Obtenido el 19 de octubre de 2020 de: http://www.scielo.org.co/scielo.php?script=sci_arttext\&pid=S0124-0064199900 0100058\&lng=en\&tlng=es.

Ursin, M. (2016). Contradictory and Intersecting Patterns of Inclusion and Exclusion of Street Youth in Salvador, Brazil. Universidad de São Paulo. 4(4) p.39-50. DOI: $10.17645 /$ si.v4i4.667

\section{CITAR COMO:}

Contreras Alfonso, A. J., Navarrete Méndez, G. D., Ortiz Urrego, T. G., \& Ávila Navarrete, V. C. (2021). Salud mental, bienestar e inclusión social en personas adultas habitantes de calle. Puriq, 3(4), 470-490. https://doi.org/10.37073/puriq.3.4.194 